H. Schulte-Wissermann

Kinderklinik, Klinikum Krefeld

\title{
Trainingsprogramm für die Weiterbildung zum Kinderarzt
}

Seit 1987 steht rekombinantes Wachstumshormon für die Behandlung von Kleinwuchs zur Verfügung. Über 25.000 Patienten sind bisher international in Studien behandelt worden, sodass sich bereits beträchtliche Erfahrungen hinsichtlich Wirkungen und Nebenwirkungen angesammelt haben. Dennoch sind noch viele Fragen zur Diagnostik und Therapie offen, besonders ob auch Kleinwuchsformen, bei denen primär kein Wachstumshormonmangel vorliegt, auch von der relativ teuren Therapie ausreichend profitieren. Um Patienten mit Kleinwuchs richtig beraten zu können, überprüfen Sie Ihren Wissensstand hinsichtlich der Therapiemöglichkeiten mit Wachstumshormon anhand der nachfolgenden Fragen. Die Therapie selbst gehört natürlich in die Hand des endokrinologischen Spezialisten.

Hinweis: Von den angebotenen Antworten kann eine, können mehrere oder alle richtig oder falsch sein.

\section{Frage 1}

Rekombinantes Wachstumshormon (WH) ist in Deutschland als Therapeutikum für folgende Kleinwuchsformen offiziell zugelassen:

A Idiopathischer WH-Mangel beim Kind

B WH-Mangel beim Erwachsenen

C Ullrich-Turner-Syndrom

D Kleinwuchs bei chronischer Niereninsuffizienz

E Intrauteriner Kleinwuchs

\section{Frage 2}

Für den idiopathischen WH-Mangel im Kindesalter gilt:

A Seltener als erworbene Form des WH-Mangels
B Kraniopharyngeom häufigste Ursache des erworbenen WH-Mangels

C Inzidenz 1:4000-10.000

D Unterschiedliche Gendefekte als Ursache

E Normalbefund im MRT des Schädels

\section{Frage 3}

Die Diagnostik des WH-Mangels erfolgt:

A Standardisiert

B In erster Linie durch Bestimmung der spontanen Basissekretion des WH

C Durch Stimulationstest mit Arginin

D Durch Stimulationstest mit Insulin

E Durch Bestimmung von IGF-1 und IGFBP-3 im Blut

\section{Frage 4}

Nach 10-jähriger Therapieerfahrung mit rekombinantem $\mathrm{WH}$ (täglich subkutan 0,4 bis 1,o IE/kg und Woche) lassen sich für Patienten mit WH-Mangel folgende Schlussfolgerungen ziehen:

A Befriedigende Endgröße bei früher Behandlung

B Befriedigende Endgröße bei Kindern, die ihr Größendefizit bereits zum Beginn der Pubertät ausgeglichen haben

C Erreichte Endgröße bei Kindern mit multiplen hypophysären Ausfällen höher als bei isoliertem WH-Mangel

D Befriedigende Endgröße bei sekundärem WH-Mangel durch Tumore im Hypophysenbereich (z. B. Kraniopharyngeom)

E Oft unbefriedigende Endgröße bei sekundärem WH-Mangel durch Medulloblastom

\section{Frage 5}

Für den Kleinwuchs bei Ullrich-TurnerSyndrom trifft zu:

A Der Kleinwuchs beruht auf einem WH-Mangel

B Die zu erwartende Erwachsenengröße lässt sich recht genau berechnen

C Durch WH-Therapie lässt sich ein Größenzugewinn von durchschnittlich $6 \mathrm{~cm}$ erreichen

D Jüngere Patienten profitieren von WH-Gaben mehr

E Mit höheren WH-Dosen lässt sich ein größerer Wachstumszugewinn erzielen

F Die Wachstumsprognose verschlechtert sich bei spätem Therapiebeginn mit Östrogen (Einleitung der Pubertät)

\section{Frage 6}

Eindeutige Nebenwirkungen der WHTherapie sind:
A Krämpfe
B Diabetes mellitus Typ I
C Diabetes mellitus Typ II
D Malignome
E Morbus Perthes

Auflösung und Kommentar der Fragen auf Seite 793. 